\title{
Authors' Reply: Safety and Efficacy of Bedside Peritoneal Dialysis Catheter Placement in the COVID-19 Era: Initial Experience at a New York City Hospital
}

\author{
Mariana Vigiola Cruz $^{1}$ (I)
}

Accepted: 8 July 2020/Published online: 29 July 2020

(C) Société Internationale de Chirurgie 2020

Dear Corresponding Author,

Thank you for your thoughtful comments regarding our article entitled, "Safety and Efficacy of Bedside Peritoneal Dialysis Catheter Placement in the COVID-19 Era: Initial Experience at a New York City Hospital" [1].

To your first point, we agree that that peritoneal dialysis proved to be a safe alternative to hemodialysis, particularly in times of limited HD resources, in personnel, machines and dialysate at the peak of our COVID-19 experience.

Secondly, we did consider the presence of SARS-CoV-2 in peritoneal fluid and indeed sent two different samples for testing. At that time and presently, we are able to obtain only a qualitative PCR test, and in both samples, the virus was undetectable. Quantitative PCR is not yet available for us to determine viral loads. While determining the presence or viral loads of SARS-CoV-2 in peritoneal fluid was not specifically a study goal in this experience, we agree this is of interest for future research.

The recommended personal protective equipment was donned per universal hospital policy for all staff working with and performing procedures on patients with COVID19. The potential infectivity of peritoneal fluid and its risk to medical staff are not yet known. Intraabdominal pressure was not significantly altered during bedside catheter placement or by the ventilatory parameters. While peritoneal dialysis patients were able to tolerate pronation, the specific ventilatory parameters in these were not a focus of this study, and the authors therefore cannot comment on this in further detail.

We agree with your final point and do hope that future research will shed light into many of the unknowns regarding SARS-CoV-2 and its effects on renal function. We do believe the indications for peritoneal dialysis should be further expanded, particularly in times and settings of resource scarcity.

\section{Reference}

1. Cruz MV, Bellorin O, Srivatana V, Afaneh C (2020) Safety and efficacy of bedside peritoneal dialysis catheter placement in the COVID-19 era: initial experience at a New York City Hospital. World J Surg 44(8):2464-2470. https://doi.org/10.1007/s00268020-05600-4

Publisher's Note Springer Nature remains neutral with regard to jurisdictional claims in published maps and institutional affiliations.
Mariana Vigiola Cruz

mav9206@med.cornell.edu

1 New York Presbyterian Hospital- Weill Cornell Medicine, 525 East 68th Street, Box 294, New York, NY 10065, USA 